\title{
Immune-Mediated Encephalopathies with an Emphasis on Paraneoplastic Encephalopathies
}

Amy A. Pruitt, M.D. ${ }^{1}$

The publisher regrets an error in the dosing units of measure in the above article in Seminars in Neurology, Volume 31, Number 2, 2011, p. 165. The correct dosing is indicated below.

Rituximab dosage should be $375 \mathrm{mg} / \mathrm{m}^{2}$ (meter squared, not millimeter squared)

Cytoxan dosage should be $750 \mathrm{mg} / \mathrm{m}^{2}$ (meter squared, not millimeter squared)

${ }^{1}$ Department of Neurology, University of Pennsylvania, Philadelphia Pennsylvania.

Address for correspondence and reprint requests: Amy A. Pruitt, M.D., Department of Neurology, University of Pennsylvania, 3400 Spruce Street, Philadelphia, PA 19104 (e-mail: pruitt@mail.med. upenn.edu).

Emerging and Controversial Issues in Neurology: Infectious
Diseases; Guest Editor, Tracey A. Cho, M.D.

Semin Neurol 2011;31:356. Copyright (C) 2011 by Thieme Medical Publishers, Inc., 333 Seventh Avenue, New York, NY 10001, USA. Tel: +1(212) 584-4662.

DOI: http://dx.doi.org/10.1055/s-0031-1291959.

ISSN 0271-8235. 